\title{
Letter
}

\section{Einstein and Rastall theories of gravitation in comparison}

\author{
F. Darabi ${ }^{1,2}$, H. Moradpour ${ }^{1}$, I. Licata ${ }^{3,4}$, Y. Heydarzade ${ }^{1,2}$, C. Corda $^{1, \text { a }}$ \\ ${ }^{1}$ Research Institute for Astronomy and Astrophysics of Maragha (RIAAM), Maragha 55134-441, Iran \\ ${ }^{2}$ Department of Physics, Azarbaijan Shahid Madani University, Tabriz, Iran \\ ${ }^{3}$ ISEM, Institute for Scientific Methodology, Palermo, PA, Italy \\ ${ }^{4}$ School of Advanced International Studies on Applied Theoretical and Non Linear Methodologies in Physics, Bari, Italy
}

Received: 16 December 2017 / Accepted: 21 December 2017 / Published online: 13 January 2018

(C) The Author(s) 2018. This article is an open access publication

\begin{abstract}
We profit by a recent paper of Visser claiming that Rastall gravity is equivalent to Einstein gravity to compare the two gravitational theories in a general way. Our conclusions are different from Visser's ones. We indeed argue that these two theories are not equivalent. In fact, Rastall theory of gravity is an "open" theory when compared to Einstein general theory of relativity. Thus, it is ready to accept the challenges of observational cosmology and quantum gravity.
\end{abstract}

The framework of extended theories of gravity [1-5] is today considered an useful and popular approach to attempt to solve the important problems of the standard model of cosmology like Dark Energy [6,7] and Dark Matter [8,9]. A key point is that all of the potential alternatives to Einstein's general theory of relativity (GTR) must be viable. This implies that alternative theories must be in agreement with the Einstein's equivalence principle, which has today a strong, unchallengeable empirical evidence [2]. The fundamental consequence is that the alternatives to Einstein gravity must be metric theories [2]. Another important point is that such alternatives must pass the solar system tests. Hence, deviations from the standard GTR must be weak [1,2].

Within the framework of extended gravity, one finds the theory proposed by Rastall in 1972 [10], which recently obtained a renewed interest in the literature [11-14]. This interest is due to some good behavior of the Rastall theory. In fact, on one hand it seems in good agreement with observational data on the Universe age and on the Hubble parameter [15]. On the other hand it may, in principle, provide an alternative description for the matter dominated era with respect to the GTR [16]. Observational data from the helium nucleosynthesis seem also in agreement with Rastall gravity [17]. Based on these observational evidences, there have been some recently studies of the various cosmic eras in the framework of Rastall gravity [18-22]. Other interesting

a e-mail: cordac.galilei@gmail.com issues are that Rastall gravity seems to do not suffer from the entropy and age problems of standard cosmology [23] and is consistent with the gravitational lensing phenomena [24,25]. Further interesting works on Rastall gravity are given by [2630] and references within.

Differently from Einstein gravity, Rastall gravity considers a non-divergence-free energy-momentum [10-30]. A different, but similar theory is the so called Curvature-matter theory of gravity [31-35]. In this theory the matter and geometry are coupled to each other in a non-minimal way [31-35]. Then, the standard energy-momentum conservation law does not work in this case too [31-35].

In a recent paper [36], Visser claimed that Rastall gravity is equivalent to Einstein gravity. In this letter, we argue that these two theories are not equivalent instead. Let us see the key points of our interpretation.

Rastall did not define a new energy-momentum tensor (EMT) in his original paper [10]. He only assumed that the ordinary conservation law is not always valid in curved spacetime and proposed a model for it. In fact, he proposed a new relation between EMT and geometry which is supported by an unknown mutual interaction between them. We think that the ordinary definition of EMT is valid in Rastall's hypothesis and there is an unknown non-minimal coupling between geometry and matter fields in Rastall gravity which should be found out in order to write its Lagrangian. Hence, the Lagrangian for the Rastall theory should be written as

$L_{E}+L_{M}+L_{i}$,

where $L_{E}$ is the standard Lagrangian of the GTR, which arises from the Ricci scalar $R, L_{M}$ is the ordinary Lagrangian of the matter fields, and $L_{i}$ is a term which includes a nonminimal coupling between geometry and matter fields. This is exactly the term which leads to the Rastall term $\left(R g_{a b}\right)$. This is an unknown term which seems to need more investigations and, in general, can be a function of $L_{E}, L_{M}, R$, 
$T, G_{a b}$ and $T_{a b}$. In summary, it seems incorrect to say that Rastall's definition of EMT is not true, as Visser has claimed in the third section of his paper [36]. We think indeed that the ordinary definition of EMT is valid in the Rastall theory, unlike the claims of Visser in [36]. In fact, we stress that violation of energy conservation has been invoked also in frameworks which are different from the Rastall gravity approach, for example in a recent attempt to achieve Dark Energy [37], while a study of classical fields in the background of a fluctuating space-time volume is considered as being a particular case of Rastall gravity [38].

We disagree with the reasoning introduced by Visser in the third section of [36]. In fact, Visser starts from a modified theory of gravity (Rastall theory) and uses the gravitational field equations to rewrite the field equations in a way in which the geometrical part is the same as that of the GTR and the matter part differs from that. Then, comparing the rewritten equations with the GTR equations, Visser gets a relation between the ordinary EMT and the EMT of Rastall gravity (Eqs. (12-15) in [36]). In other words, Visser implicitly assumes a priori that the Rastall equation, i.e. Eq. (2) in [36], is equivalent to the standard Einstein's equation, i.e. Eq. (9) in [36]. As a consequence, all Visser's derivations in the third Section of [36] are simple rearrangements, but, in our opinion, such rearrangements have no physical meaning being due to Visser's a priori assumption that Eqs. (2) and (9) in [36] are equivalent. They are not equivalent instead. Let us see this point in more detail. The key point is that in [36] Visser has put a new tensor, labelled $\left[T_{R}\right]_{a b}$ in the right hand side of his Eq. (2), whereas if one rearranges the terms in Eq. (1) in Rastall's original paper [10], one sees that it has the form

$G_{a b}=\kappa T_{a b}^{(m)}-\kappa \lambda R g_{a b}$,

where $T_{a b}^{(m)} \neq\left[T_{R}\right]_{a b}$ is the ordinary EMT. Now, if one uses the normalization of Visser $(\kappa \lambda=\lambda / 4)$, one finds

$G_{a b}+\frac{\lambda}{4} R g_{a b}=\kappa T_{a b}^{(m)}$

Comparing this equation (originally obtained by Rastall in [10] without Visser's normalization $\kappa \lambda=\lambda / 4$ ) with Eq. (2) of Visser's paper [36] which is

$G_{a b}+\frac{\lambda}{4} R g_{a b}=\kappa\left[T_{R}\right]_{a b}$,

one realizes that Visser has erroneously put $\left[T_{R}\right]_{a b}$ instead of $T_{a b}^{(m)}$ in the right hand side of Eq. (0.3). If he would have written the correct $T_{a b}^{(m)}$, then he would have obtained the correct Eqs. (8) and (9) in [36], in which $\left[T_{R}\right]_{a b}$ is replaced by $T_{a b}^{(m)}$. Thus, he would have obtained $G_{a b}=\kappa\left[T_{R}\right]_{a b}$ instead of $G_{a b}=\kappa T_{a b}^{(m)}$. In fact, the original version of Rastall gravity [10] leads to the modified equation

$G_{a b}=\kappa T^{(m)} R_{a b}$

(where $\left.\left[T_{R}\right]_{a b}=\left(T_{a b}^{(m)}+\frac{1}{4} \frac{\lambda}{1-\lambda} T^{(m)} g_{a b}\right)\right)$ and NOT

$G_{a b}=k T_{a b}^{(m)}$,

(where $\left.T_{a b}^{(m)}=\left(\left[T_{R}\right]_{a b}+\frac{1}{4} \frac{\lambda}{1-\lambda}\left[T_{R}\right] g_{a b}\right)\right)$ as Visser has obtained. Due to the Bianchi identity one finds

$\nabla^{b} G_{a b}=0$

and, in turn,

$\nabla^{b}\left[T_{R}\right]_{a b}=0$

which is NOT $\nabla^{b} T_{a b}^{(m)}=0$. Now, Eq. (0.8) is equivalent to

$\nabla^{b} T_{a b}^{(m)}=-\frac{1}{4} \frac{\lambda}{1-\lambda} \nabla^{b} T^{(m)} g_{a b}$

or

$\nabla^{b} T_{a b}^{(m)}=\frac{1}{4} \lambda \nabla^{b} R g_{a b}$

which accounts for the non-conservation of Einstein's EMT $T_{a b}^{(m)}$ according to the original idea of Rastall in [10]. Hence, as the source of $\nabla^{b} T_{a b}^{(m)}$ is the 4-dimensional geometry itself (namely $\nabla^{b} R g_{a b}$ ), the consequence is that Rastall gravity is drastically different from Einstein gravity in 4-dimensions.

Some criticisms on Rastall gravity (and on other nonconservative gravitational theories) have also been raised in the early paper [44]. In fact, the discussion in [44] is almost the same as the one in [36] and a bit more detailed. One verbatim reads in [44]: "The question which remains is how to construct the stress-energy tensor out of the matter fields of the real world. Since $S_{\mu \nu}$ is always exactly conserved, and the definition of $S_{\mu \nu}$ in terms of $T_{\mu \nu}$ (3), is independent of space-time curvature, this remaining question is really a question about the conservation of stress-energy in special relativity, and not an alternative theory of gravity at all." The quantities $S_{\mu \nu}$ and $T_{\mu \nu}$ in [44] are the same quantities that we labelled $\left[T_{R}\right]_{a b}$ and $T_{a b}^{(m)}$ in this letter, respectively. Exactly like in [36], $S_{\mu \nu}$ is defined as being "a new stressenergy tensor" by the authors of [44]. There is a key point in the sentence in [44] "the definition of $S_{\mu \nu}$ in terms of $T_{\mu v}(3)$, is independent of space-time curvature" which have led the authors of [44] (and Visser in [36]) to suppose that there is essentially no non-minimal coupling of matter with geometry. The point is that, there is certainly a non-minimal coupling present in the coupling $\lambda / 1-4 \lambda$ in Eq. (3) of [44]. 
This coupling comes exactly from the trace of the Rastall field equation. The apparent absence of the Ricci scalar in Eq. (3) of [44] does not mean that it is independent of the space-time curvature. In fact, the definition of $S_{\mu \nu}$ in terms of $T_{\mu \nu}$ (3) in Eq. (3) of [44] IS DEPENDENT on the space-time curvature indirectly through the trace equation. Without such dependence and non-minimal coupling between the Ricci scalar $R$ and the trace of the EMT, the $\lambda / 1-4 \lambda$ in Eq. (3) of [44] would not exist and so $S_{\mu \nu}$ would not satisfy the conservation equation. Such a non-minimal coupling is crucial for the covariant conservation of $S_{\mu \nu}$.

Visser also argues in [36] that since the ordinary EMT differs from the obtained EMT, the Rastall's definition of EMT is incorrect. We think that this argument should be not true. If it is true, then one can generalize his recipe to all of the modified gravity theories and concludes that all of them are incorrect and are special rearrangements of the GTR, which is, of course, not true. Let us consider, as a simple example, $f(R)$ gravity $[3,4]$. In that case, the field equations are [4]

$$
\begin{aligned}
G_{a b}= & \frac{1}{f^{\prime}(R)}\left\{\frac{1}{2} g_{a b}\left[f(R)-f^{\prime}(R) R\right]\right. \\
& \left.+f^{\prime}(R)_{; a ; b}-g_{a b} \square f^{\prime}(R)\right\}+\kappa T_{a b}^{(m)},
\end{aligned}
$$

where $\kappa$ is the gravitational coupling constant. Defining the curvature fluid EMT as [4]

$$
\begin{aligned}
T_{a b}^{(c)} \equiv & \frac{1}{\kappa f^{\prime}(R)}\left\{\frac{1}{2} g_{a b}\left[f(R)-f^{\prime}(R) R\right]\right. \\
& \left.+f^{\prime}(R)_{; a ; b}-g_{a b} \square f^{\prime}(R)\right\},
\end{aligned}
$$

one immediately rewrites Eq. (0.11) as

$G_{a b}=\kappa T_{a b}^{(c)}+\kappa T_{a b}^{(m)}$

Now, if one introduces

$$
T_{a b}^{(\text {total })} \equiv\left(T_{a b}^{(m)}+T_{a b}^{(c)}\right)
$$

the gravitational field equations in $f(R)$ gravity obtain the Einsteinian form

$$
G_{a b}=\kappa T_{a b}^{(\text {total })}
$$

Equation (0.15) is formally equal to the traditional field equations of the GTR, which are

$G_{a b}=\kappa T_{a b}^{(m)}$

but, of course, this does not mean that the GTR is equivalent to $f(R)$ gravity. In fact, such an equivalence exists only in the particular case $f(R)=R$, as it is well known.
It is also important to stress that two different modified theories may have different vacuum solutions since there may be some different degrees of freedom associated to each of these theories. Reversely, if two theories have same vacuum solutions, one can not deduce any result for the equivalence of those theories. This is because the vacuum solution is just a specific solution which one can not read all the degrees of freedom of the underlying theory from that. For example, in [39] it has been shown that there are vacuum solutions on a $4 \mathrm{D}$ brane which are completely different than the vacuum solutions in the GTR. The same happens for the brane solutions with non-vanishing bulk Weyl tensor.

We find remarkable differences between Einstein and Rastall gravities in cosmological solutions, as it has been recently shown in [40]. In addition, we emphasize that, from the cosmological point of view, Rastall hypothesis (mutual interaction between geometry and matter fields) cannot describe Dark Energy and one need to consider a Dark Energy-like source, just the same as Einstein GTR [21,40]. In addition, one may describe the current era, by generalizing the Rastall hypothesis and without using a Dark Energy-like source [41].

Finally, non-equivalence between Einstein and Rastall theories of gravity was early stressed in an old but interesting paper of Smalley [42].

For the sake of completeness, we take the chance to recall some further interesting issue and some weakness of Rastall gravity. From the point of view of Mach principle, Rastall gravity seems more "Machian" than Einstein gravity [43]. Till now, physicists interacting with Rastall gravity have at most focused on its cosmological features. Thus, one can consider as being a weakness of the Rastall framework the difficulty to relate the accelerating expansion of the Universe to the Rastall interaction. On the other hand, this is also a well known problem of the GTR. Another weakness is that the Rastall Lagrangian is not completely known, despite a first approach on this issue has been recently developed in [38]. In fact, we think that Rastall theory of gravity needs more studies to become familiar with all its aspects.

\section{Conclusion remarks}

In this letter, we profited by the recent paper of Visser [36], where it is claimed that Rastall gravity is equivalent to Einstein gravity, to compare the two gravitational theories in a general way. The conclusions of the current work are different from the ones in [36]. We have indeed raised various issues showing that these two theories are not equivalent. As a consequence, we can consider Rastall gravity as being an "open" theory compared to Einstein gravity. Hence, Rastall theory of gravity seems to be an extended theory of gravity 
ready to accept the challenges of observational cosmology and quantum gravity.

Acknowledgements This letter has been supported financially by the Research Institute for Astronomy and Astrophysics of Maragha (RIAAM), Project Number 1/5440-15.

Open Access This article is distributed under the terms of the Creative Commons Attribution 4.0 International License (http://creativecomm ons.org/licenses/by/4.0/), which permits unrestricted use, distribution, and reproduction in any medium, provided you give appropriate credit to the original author(s) and the source, provide a link to the Creative Commons license, and indicate if changes were made.

Funded by SCOAP ${ }^{3}$.

\section{References}

1. C. Corda, Int. J. Mod. Phys. D 18, 2275 (2009)

2. C.M. Will, Living Rev. Relativ. 17, 4 (2014)

3. S. Nojiri, S.D. Odintsov, Phys. Rep. 505, 59 (2011)

4. S. Capozziello, M. De Laurentis, Phys. Rep. 509, 167 (2011)

5. S. Nojiri, S.D. Odintsov, V.K. Oikonomou, Phys. Rept. 692, 1 (2017)

6. A.G. Riess et al., Astron. J. 116, 1009 (1998)

7. A.G. Riess et al., Astrophys. J. 560, 49 (2001)

8. D. Clowe et al., Astrophys. J. 648, L109 (2006)

9. J.F. Navarro, C.S. Frenk, S.D.M. White, Astrophys. J. 490, 493 (1997)

10. P. Rastall, Phys. Rev. D 6, 3357 (1972)

11. H. Moradpour, I.G. Salako, Adv. High Energy Phys. 2016, 3492796 (2016)

12. F.-F. Yuan, P. Huang, Class. Quantum Gravity 34, 077001 (2017)

13. H. Moradpour, Phys. Lett. B 757, 187 (2016). final version in arXiv: 1601.04529

14. A.M. Oliveira, H.E.S. Velten, J.C. Fabris, Phys. Rev. D 92, 044020 (2015)

15. A.S. Al-Rawaf, O.M. Taha, Phys. Lett. B 366, 69 (1996)
16. A.S. Al-Rawaf, O.M. Taha, Gen. Relat. Gravit. 28, 935 (1996)

17. A.S. Al-Rawaf, Int. J. Mod. Phys. D 14, 1941 (2005)

18. V. Majernik, Gen. Relat. Gravit. 35, 1007 (2003)

19. A.I. Arbab, JCAP 05, 008 (2003)

20. A.-M.M. Abdel-Rahman, Ihab F. Riad, Astron. J. 134, 1391 (2007)

21. C.E.M. Batista, M.H. Daouda, J.C. Fabris, O.F. Piattella, D.C. Rodrigues, Phys. Rev. D 85, 084008 (2012)

22. J.C. Fabris, O.F. Piattella, D.C. Rodrigues, C.E.M. Batista, M.H. Daouda, Int. J. Mod. Phys. Conf. Ser. 18, 67 (2012)

23. J.C. Fabris, R. Kerner, J. Tossa, Int. J. Mod. Phys. D 9, 111 (2000)

24. A.-M.M. Abdel-Rahman, Astrophys. Space Sci. 278, 383 (2001)

25. A.-M.M. Abdel-Rahman, M.H.A. Hashim, Astrophys. Space Sci. 298, $519(2005)$

26. A.F. Santos, S.C. Ulhoa, Mod. Phys. Lett. A 30, 1550039 (2015)

27. Y. Heydarzade, F. Darabi, Phys. Lett. B 771, 365 (2017)

28. Y. Heydarzade, H. Moradpour, F. Darabi, Can. J. Phys. 95, 1253 (2017)

29. H. Moradpour, N. Sadeghnezhad, S.H. Hendi, Can. J. Phys. 95, 1257 (2017)

30. I. Licata, H. Moradpour, C. Corda, Int. J. Geom. Methods Mod. Phys. 14, 1730003 (2017)

31. G. Allemandi, A. Borowiec, M. Francaviglia, S.D. Odintsov, Phys. Rev. D 72, 063505 (2005)

32. S. Nojiri, S.D. Odintsov, Phys. Lett. B 599, 137 (2004)

33. T. Koivisto, Class. Quantum Gravit. 23, 4289 (2006)

34. O. Bertolami, C.G. Boehmer, T. Harko, F.S.N. Lobo, Phys. Rev. D 75, 104016 (2007)

35. T. Harko, F.S.N. Lobo, Galaxies 2, 410 (2014)

36. M. Visser, arXiv:1711.11500

37. T. Josset, A. Perez, Phys. Rev. Lett. 118, 021102 (2017)

38. V. Dzhunushaliev, H. Quevedo, Gravit. Cosmol. 23, 280 (2017)

39. Y. Heydarzade, H. Hadi, C. Corda, F. Darabi, Phys. Lett. B 776, 457 (2018)

40. H. Moradpour, A. Bonilla, E.M.C. Abreu, J.A. Neto, Phys. Rev. D 96, 123504 (2017)

41. H. Moradpour, Y. Heydarzade, F. Darabi, I.G. Salako, Eur. Phys. J. C 77, 259 (2017)

42. L.L. Smalley, J. Phys. A Math. Gen. 16, 2179 (1983)

43. V. Majernik, L. Richterek, arXiv:gr-qc/0610070 (2006)

44. L. Lindblom, W.A. Hiscock, J. Phys. A Math. Gen. 15, 1827 (1982) 